\title{
Editorial
}

\section{Mathematical Modeling of Heat and Mass Transfer in Energy Science and Engineering 2014}

\author{
Zhijun Zhang, ${ }^{1}$ Hua-Shu Dou, ${ }^{2}$ Ireneusz Zbicinski, ${ }^{3}$ Zhonghua Wu, ${ }^{4}$ and Jun Liu ${ }^{5}$ \\ ${ }^{1}$ School of Mechanical Engineering and Automation, Northeastern University, Shenyang 110004, China \\ ${ }^{2}$ Faculty of Mechanical Engineering and Automation, Zhejiang Sci-Tech University, Hangzhou, Zhejiang 310018, China \\ ${ }^{3}$ Faculty of Process and Environmental Engineering, Technical University of Lodz, 90-924 Lodz, Poland \\ ${ }^{4}$ Institute of Drying and Dewatering, College of Mechanical Engineering, Tianjin University of Science and Technology, \\ 1038 Daguan Road, Hexi District, Tianjin 300222, China \\ ${ }^{5}$ Institute of Biology and Chemistry, Shenyang University, Shenyang 110044, China
}

Correspondence should be addressed to Zhijun Zhang; zhjzhang@mail.neu.edu.cn

Received 29 September 2014; Accepted 29 September 2014

Copyright ( 2015 Zhijun Zhang et al. This is an open access article distributed under the Creative Commons Attribution License, which permits unrestricted use, distribution, and reproduction in any medium, provided the original work is properly cited.

Heat and mass transfer process is the basis of energy research from the boiler, gas turbine to fuel cell, solar power. It is the need of energy conversion and transfer process. The heat and mass transfer process mainly include the following aspects: thermodynamics, power and fluid machinery, heat and mass transfer, combustion, and multiphase flow. The idea of this special issue is to consider the study and applications of mathematical modeling method on energy science and technology.

This special issue contains 27 papers, the contents of which are summarized as follows.

The paper entitled "A numerical study of natural convection heat transfer in fin ribbed radiator" by H.-S. Dou et al. numerically investigates the physical mechanism of flow instability and heat transfer of natural convection in a cavity fixed with fin arrays.

In "A differential-algebraic model for the once-through steam generator of MHTGR-based multi-modular nuclear plants" by Z. Dong, based upon the conservation laws of mass, energy, and momentum, a new differential-algebraic model for the OTSGs of the MHTGR based multimodular nuclear plants is given.

In "Numerical investigations of the effect of nonlinear quadratic pressure gradient term on a moving boundary problem of radial flow in low-permeable reservoirs with threshold pressure gradient" by W. Liu and J. Yao, based on these concerns, in consideration of the QPGT, a moving boundary model of radial flow in low-permeable reservoirs with the TPG for the case of a constant flow rate at the inner boundary is constructed.

In "Numerical study of buoyancy convection of air under permanent magnetic field and xomparison with that under gravity field" by K. Song et al., magneto thermal free convection of air in a square enclosure under a nonuniform magnetic field provided by a permanent neodymium-ironboron magnet is numerically studied.

In "System model of heat and mass transfer process for mobile solvent vapor phase drying equipment" by S. Zhang et al., on the basis of necessary simplification and assumption for MVPD equipment and process, a heat and mass transfer mathematical model including 40 mathematical equations is established, which represents completely thermodynamics laws of phase change and transport process of solvent, water, and air in MVPD technological processes and describes in detail the quantitative relationship among important physical quantities such as temperature, pressure, and flux in key equipment units and process.

The paper entitled "Research on the impact of wind angles on the residential building energy consumption" by Z. Huifen et al., combined with natural ventilation under various wind 
angles, gives the best recommended solution of building direction in Shenyang.

In "Local fractional Laplace variational iteration method for nonhomogeneous heat equations arising in fractal heat flow" by S. Xu et al., the approximate solutions are nondifferentiable functions and their plots are also given to show the accuracy and efficiency to implement the previous method.

In "Numerical simulation and experimental research on coal ash collecting and grading system" by Y. Xie at al., focused on single coal ash particle, Matlab software was used to simulate the force conditions and separation parameters of various diameter coal ash particles in airflow. Fluent software was used to simulate the nozzle fluidization domain shape and to determine optimal jet flux.

In "Simulation of microstructure during laser rapid forming solidification based on cellular automaton" by Z. Wang et al., the grain microstructure of molten pool during the solidification of TC4 titanium alloy in the single point laser cladding was investigated based on the CAFE model which is the cellular automaton (CA) coupled with the finite element (FE) method.

In "Application of CFD, Taguchi method, and ANOVA technique to optimize combustion and emissions in a light duty diesel engine" by S. Xiao et al., in order to understand the combined effect of EGR rate, pilot fuel quantity, and main injection timing on the $\mathrm{NO}_{x}$ (oxides of nitrogen), soot, and ISFC (indicated specific fuel consumption), CFD (computational fluid dynamics) simulation together with the Taguchi method and the ANOVA (analysis of variance) technique was applied as an effective research tool.

In "Mathematical simulation of heat and mass transfer processes at the ignition of liquid fuel by concentrated flux of radiation" by $\mathrm{O}$. V. Vysokomornaya et al., the physical and forecasting mathematical models of heat and mass transfer with phase transformations and chemical reactions under heating and following ignition of typical liquid fuel by using concentrated flow of radiation were developed.

In "Heat and mass transfer of droplet vacuum freezing process based on dynamic mesh" by L. Zhao et al., the initial droplet diameter, initial droplet temperature, and vacuum chamber pressure effect are studied.

In "Entropy generation analysis of power-law nonNewtonian fluid flow caused by micropatterned moving surface" by M. H. Yazdi et al., the first and second law analyses of power-law non-Newtonian flow over embedded open parallel microchannels within micropatterned permeable continuous moving surface are examined at prescribed surface temperature.

In "Numerical investigation on a prototype centrifugal pump subjected to fluctuating rotational speed" by Y.-L. Zhang et al., in order to study the transient response characteristic of a prototype centrifugal pump subjected to fluctuating rotational speed, a closed-loop pipe system including the pump is built to accomplish unsteady flow calculations in which the boundary conditions at the inlet and the outlet of the pump are not required to be set.

In "Numerical and experimental research of heat and mass transfer at the heterogeneous system ignition by local energy source with limited heat content" by D. O. Glushkov et al., numerical and experimental investigations were executed for determination of macroscopic regularities of heat and mass transfer processes under the conditions of the phase transformations and chemical reaction at the ignition of vapors coming from fabrics impregnated by combustible liquid into oxidant area at the local power supply.

The paper entitled "An improved dispatch strategy of a grid-connected hybrid energy system with high penetration level of renewable energy" by Y. Zhang et al. focuses on the fluctuation alleviation and power quality improvement of grid-connected HES with high penetration level of RES.

In "Analysis of combined power and refrigeration generation using the carbon dioxide thermodynamic cycle to recover the waste heat of an internal combustion engine" by $\mathrm{S}$. Wang et al., a novel thermodynamic system is proposed to recover the waste heat of an internal combustion engine (ICE) by integrating the transcritical carbon dioxide $\left(\mathrm{CO}_{2}\right)$ refrigeration cycle with the supercritical $\mathrm{CO}_{2}$ power cycle, and eight kinds of integration schemes are developed.

In "Mathematical modeling of eddy-current loss for a new induction heating device" by $\mathrm{H}$. Du et al., this device can convert mechanical energy into heat energy by utilizing eddy currents, which are induced by rotating permanent magnets. A mathematical model is established for estimating eddycurrent loss of the device.

In "Energy loss in pulse detonation engine due to fuel viscosity" by $\mathrm{W}$. Hu et al., to analyze the energy loss in the pulse detonation engine (PDE) due to the viscosity of the fuel, the energy loss in the Burgers model excited by periodic impulses is investigated based on the generalized multisymplectic method in this paper.

The paper entitled "Pore network analysis of zone model for porous media drying" by Y. Yuejin et al. fused the physical parameters of porous media, such as porosity, pore mean diameter, and pore size distribution into the model parameters, and a sand bed drying experiment was conducted to verify the validity of this model.

In "Model-based water wall fault detection and diagnosis of FBC boiler using strong tracking filter" by L. Sun et al., a model-based approach is presented to estimate internal states and heat transfer coefficient dually from the noisy measurable outputs.

In "Application of D-S evidence fusion method in the fault detection of temperature sensor" by Z. Dou et al., based on the idea of information fusion and the requirements of D-S evidence method, a D-S evidence fusion structure with two layers was introduced to detect the temperature sensor fault in drying process.

The paper entitled "Mixed convection unsteady stagnation-point flow towards a stretching sheet with slip effects" by $\mathrm{H}$. Chen studies the unsteady mixed convection flow of an incompressible viscous fluid about a stagnation point on a stretching sheet in presence of velocity and thermal slips.

The paper entitled "Effects of wall shear stress on MHD conjugate flow over an inclined plate in a porous medium with ramped wall Temperature" by A. Khan et al. investigates the effects of an arbitrary wall shear stress on unsteady magnetohydrodynamic (MHD) flow of a Newtonian fluid with conjugate effects of heat and mass transfer. 
In "An optimization model based on electric power generation in steel industry" by J. Liu and J. Cai, under the premise of ensuring the stability of energy supply and the normal production safety, the mathematical programming method and the dynamic mathematical optimization model were used to set up the surplus gas in the optimal allocation among the buffer users and steam production dispatching for the production equipment.

The paper entitled "Identification of shaft centerline orbit for wind power units based on Hopfield neural network improved by simulated annealing" by K. Ren and J. Qu presents the diagnosis of the orbit as follows: acquire characters of orbit by the affine invariant moments, take this as the characteristic parameters of neural networks to construct the identification model, and utilize simulated annealing (SA) algorithm to optimize the weights matrix of Hopfield neural network, and then some typical faults were selected as examples to identify.

Zhijun Zhang

Hua-Shu Dou

Ireneusz Zbicinski

Zhonghua Wu

Jun Liu 


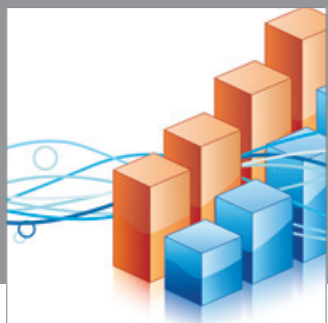

Advances in

Operations Research

mansans

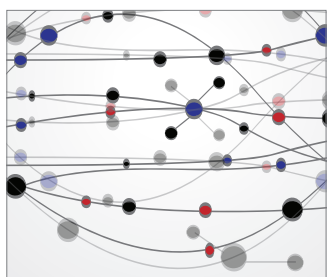

The Scientific World Journal
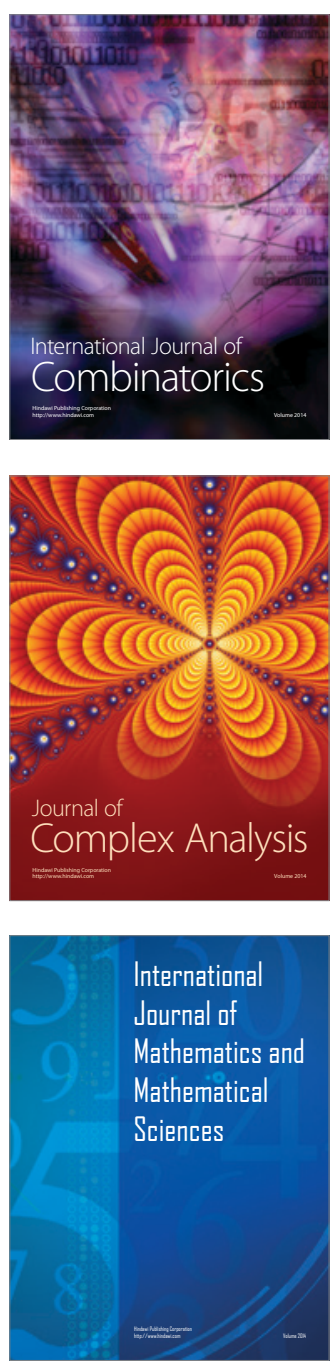
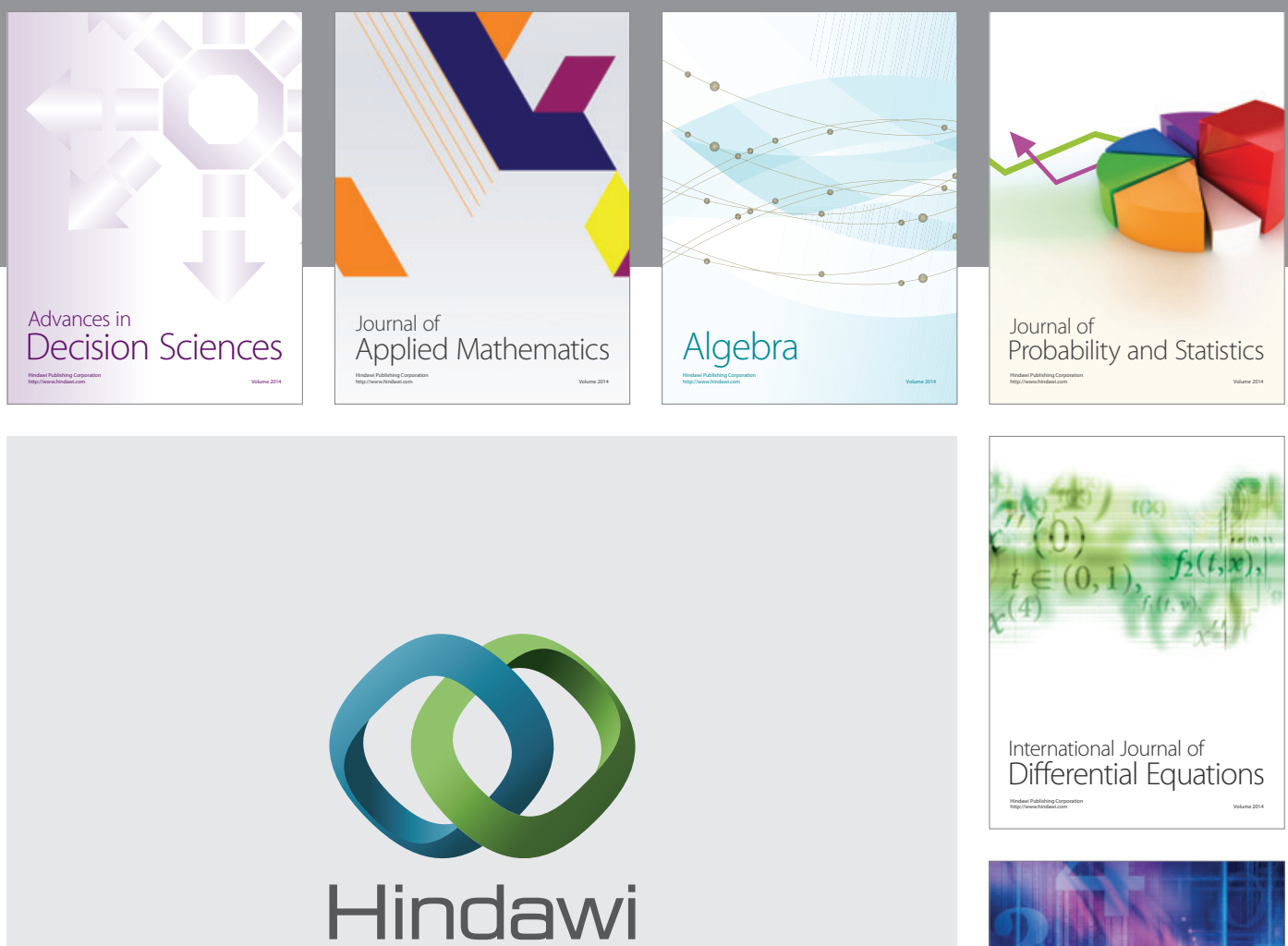

Submit your manuscripts at http://www.hindawi.com
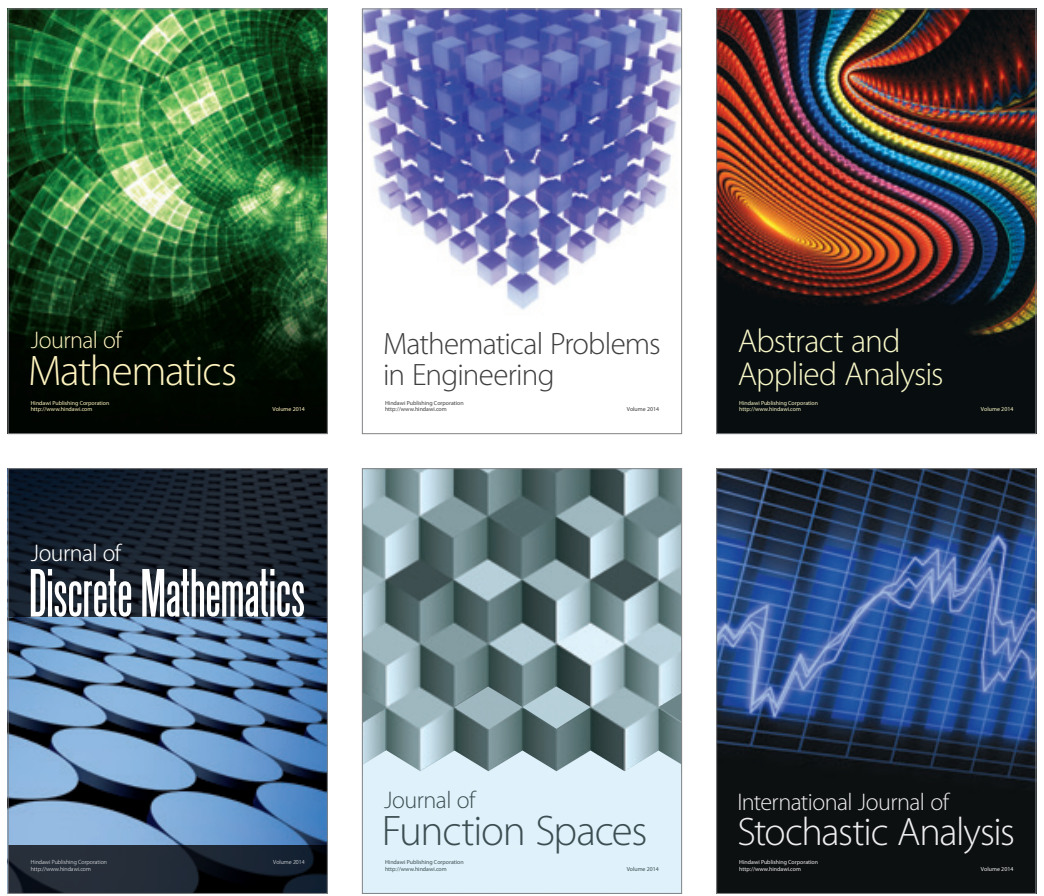

Journal of

Function Spaces

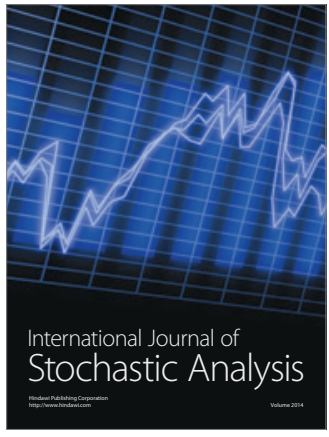

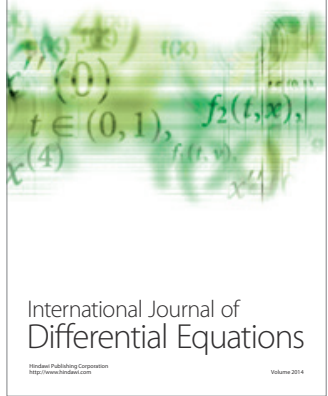
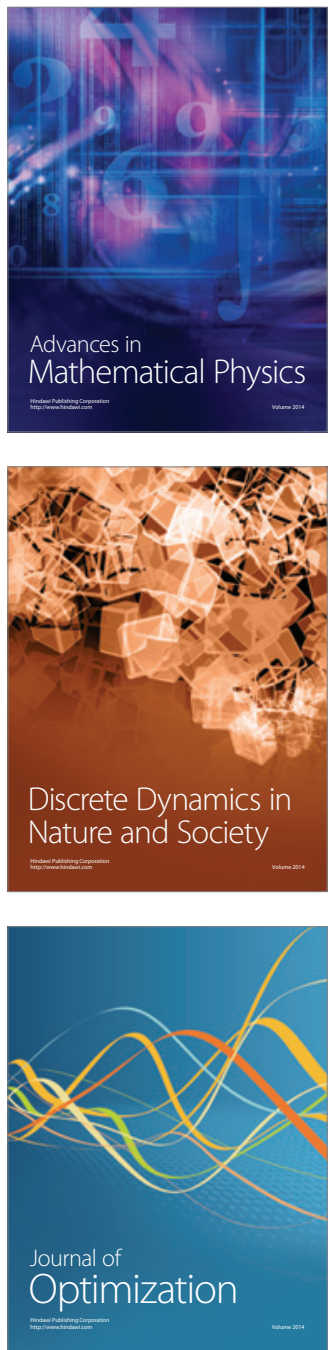